\title{
Dissociations among tasks involving inhibition: A single-case study
}

\author{
A. CRIS HAMILTON and RANDI C. MARTIN \\ Rice University, Houston, Texas
}

\begin{abstract}
Recent theories of working memory have emphasized the role of inhibition in suppressing irrelevant information. Moreover, psychometric studies have reported that several inhibition tasks with very diverse requirements load on a single inhibition factor. A patient with left inferior frontal damage, Patient M.L., previously reported to have a semantic short-term memory deficit (R. C. Martin \& He, 2004), showed evidence of difficulty with inhibition on short-term memory tasks. We investigated whether he would show evidence of inhibition difficulty on two verbal tasks (a Stroop task and a recent-negatives task) and two nonverbal tasks (a nonverbal spatial Stroop task and an antisaccade task). M.L. was impaired on both verbal tasks but performed normally on the nonverbal tasks. M.L.'s data also represent a dissociation between Stroop and antisaccade performance, two tasks that load on a single factor in factor-analytic studies. The implications of these data for theories of inhibition and executive function are discussed.
\end{abstract}

Executive function is associated with complex planning and the ability to organize behavior. A core component of executive processes is inhibition - a term referring to the suppression of irrelevant information and overlearned or prepotent responses. Tasks commonly associated with inhibition measure a seemingly diverse set of abilities, and one might question whether all such tasks measure the same cognitive processes. For example, it is not obvious that the processes necessary to inhibit reflexive eye movements to sudden stimulus onsets in an antisaccade task are the same as those necessary to inhibit the tendency to name the word, rather than the color, in the Stroop task. Rather than positing a global inhibitory process, it may be necessary to further fractionate the processes involved in inhibition. In the present study, we begin to address these issues concerning the nature of inhibitory processes by examining whether these processes dissociate in a braindamaged patient with left inferior frontal damage.

Previous research with normal subjects has suggested that several different tasks involving inhibition do share common processes. In an effort to delineate the components of executive function, Miyake et al. (2000) collected data from a dozen tasks commonly assumed to involve executive processes and performed a latent variable analysis. This analysis yielded three factors, which Miyake et al. identified as shifting, updating, and inhibition. According to Miyake et al., shifting represents the ability to shift

This research was supported by NIH Grant DC-00218 to R.C.M. at Rice University. The authors acknowledge the assistance of Meredith Knight and Jill Henderson in collecting control data for this project and thank Naomi Friedman and Akira Miyake for providing the experimental tasks. Some of these data were presented at the 40th Annual Meeting of the Academy of Aphasia. Correspondence concerning this article should be addressed to R. C. Martin, Psychology Department, MS-25, Rice University, P.O. Box 1892, Houston, TX 77251 (e-mail: rmartin@rice.edu). cognitive control between different tasks or routines. Updating is the ability to update and monitor representations in working memory (WM). Finally, inhibition is described as the ability to inhibit irrelevant information or prepotent responses. Tasks that loaded on the inhibition factor included the antisaccade task, the stop signal task, and the Stroop task. Miyake et al. made no explicit predictions as to whether these factors may be associated with distinct and separable neural substrates. However, one might predict that a patient who performed poorly on one inhibition task would show a deficit on other tasks loading on the inhibition factor but would not necessarily have difficulty with tasks loading on the other factors. In fact, Miyake et al. recommended that their results be carefully evaluated in neuropsychological populations in order to provide converging evidence. The present study takes this approach.

In addition to its fundamental role in executive function, inhibition and the ability to resist interference have been the subject of an increasing number of studies in the memory literature. Recent theories of WM have emphasized resistance to interference as accounting for much of the individual variability in memory performance among normal individuals (see Rosen \& Engle, 1997; Whitney, Arnett, Driver, \& Budd, 2001; Zacks \& Hasher, 1994). Among college age subjects, Engle and colleagues have also demonstrated a relationship between WM capacity and susceptibility to interference in a number of different paradigms (Rosen \& Engle, 1997, 1998). In the literature on aging, poorer performance for older adults on WM tasks has been attributed to difficulty inhibiting irrelevant information. Studies by Hasher and colleagues (Chiappe, Hasher, \& Siegel, 2000; May, Hasher, \& Kane, 1999) have shown that the typically poorer WM performance of older adults than of younger adults could be eliminated if 
procedures that minimized proactive interference were used. Similarly, others have attempted to use susceptibility to proactive interference-specifically, a susceptibility to intrusions of previously presented material - as a behavioral marker of various neurological deficits that serve to undermine memory (Rouleau, Imbault, Laframboise, \& Bédard, 2001).

Relevant to the question of WM capacity and susceptibility to interference is a series of recent studies in which a probe task (formulated by Monsell, 1978) has been employed to induce proactive interference. This task, subsequently referred to as the recent-negatives task, consists of some trials in which the probe item matches an item in the preceding list, but not on the current list. These studies (D'Esposito, Postle, Jonides, \& Smith, 1999; Jonides et al., 2000; Jonides, Smith, Marshuetz, Koeppe, \& Reuter-Lorenz, 1998) have identified unique brain areas involved in overcoming proactive interference. Specifically, Jonides et al. (1998) have demonstrated increased activation in the left inferior prefrontal cortex, in the region of Brodmann's area (BA) 45. This activation in BA 45 is uniquely associated with items presumably affected by proactive interference. Moreover, Jonides et al. (2000) have reported that elderly subjects show greater susceptibility to proactive interference when compared with younger adults and a corresponding absence of cortical activation in BA 45 during trials that promote such interference. In addition, Thompson-Schill et al. (2002) have demonstrated that a patient with damage to BA 45 showed exaggerated effects of proactive interference on this task.

A very different inhibition paradigm reported to be associated with WM was first reported by Roberts, Hager, and Heron (1994) and has been employed by Kane, Bleckley, Conway, and Engle (2001). This paradigm is notable because it employs a nonverbal task with no obvious memory requirements. Subjects are required to maintain fixation and are then presented with a brief distractor appearing in the periphery of the display. The subjects must inhibit the tendency to make a reflexive saccade to this distractor in order to successfully make an eye movement to a brief target presented on the opposite side. Reported correlations between WM capacity and performance on the antisaccade task have prompted many to propose a role for attention in WM capacity (see Kane et al., 2001; Mitchell, Macrae, \& Gilchrist, 2002; Roberts et al., 1994). Specifically, WM is thought to involve an ability to inhibit prepotent responses.

Given the reported relationships among inhibition and WM performance, we have asked whether patients with short-term memory (STM) deficits will demonstrate deficits on measures of inhibition. Although our research program has previously emphasized the role of language representations in STM (e.g., R. C. Martin \& Lesch, 1996), we have recently become interested in a possible role for inhibition in conceptualizing some types of STM. Next, we will describe our model of STM organization.
Distinct patterns of patient performance on various tasks involving STM support a dissociation between semantic and phonological capacities in STM. For example, patients with semantic STM deficits typically demonstrate no advantage of words relative to nonwords (no lexicality effect), whereas patients with phonological STM deficits fail to demonstrate normal phonological similarity effects. Patients with semantic STM deficits perform better on a rhyme probe task, relative to a category probe task, whereas phonological patients are better on the category probe than on the rhyme probe task (R. C. Martin, Shelton, \& Yaffee, 1994). It is believed that patients with semantic STM deficits are unable to accurately maintain lexical-semantic representations in STM and must therefore rely on phonological representations. On the other hand, cases with phonological STM deficits are unable to adequately maintain phonological information and depend upon lexical-semantic information during recall. Related patterns for brain-damaged patients have been reported by N. Martin and Saffran (1997).

Of relevance to the present study, R. C. Martin and Lesch (1996) reported a particularly interesting observation in patients with semantic STM deficits. These patients made numerous intrusions of previously presented items during serial recall tasks. This effect is surprising given that, if patients' STM deficits are attributed to reduced capacity or an abnormally fast decay rate, one might expect fewer intrusions from previous lists. Interestingly, this paradoxical effect of excessive intrusions in patients with greatly limited STM has been observed only in cases of semantic STM deficits. Here, we will address these intrusions as an apparent failure to inhibit the processing of previously presented items, which produces exaggerated effects of proactive interference.

Anatomically, semantic STM deficits are associated with lesions involving inferior frontal areas (R. C. Martin \& Freedman, 2001; Romani \& Martin, 1999). This observation is intriguing, given recent fMRI data implicating inferior frontal areas with inhibitory functions, such as semantic selection (Thompson-Schill, D'Esposito, Aguirre, $\&$ Farah, 1997) and resolution of proactive interference (Jonides et al., 1998). Moreover, other interference effects possibly attributable to failures of inhibition have been reported; for example, Freedman, Martin, and Biegler (2004) reported that patients with semantic STM deficits demonstrated greatly exaggerated interference effects when producing a conjoined noun phrase to describe two semantically related items, as compared with two unrelated items. These observations implicate a possible role for impaired inhibitory function in semantic STM deficits.

Given the prominent role of inhibition in many contemporary models of WM and the aforementioned patient performance suggesting deficits of inhibitory function, in the present study, we examined the performance of a semantic STM patient on tasks reported to be associated with inhibition. We included two tasks identified by Miyake et al. (2000) as loading on an inhibition factor- 
the standard Stroop task and the antisaccade task. We also employed a probe task designed to elicit proactive interference (Jonides et al., 1998; Monsell, 1978). Finally, we included a nonverbal spatial Stroop task as a nonverbal analogue to the Stroop task.

In the present study, we attempted to address several questions. First, would a patient who showed a semantic STM deficit and intrusions of previous list items on serial recall show a deficit on inhibition tasks not involving STM? If so, would this deficit on inhibition tasks include a deficit on the antisaccade task, a nonverbal task that has been associated with WM function? Second, would such a patient show an exaggerated effect on the recent-negatives task that manipulates proactive interference? Third, would the patient generally show a difference in performance between verbal and nonverbal inhibition tasks? Given that the previously reported deficits of this patient are language related (see, e.g., R. C. Martin \& He, 2004) and that his lesion is in the left hemisphere, it is possible that any noted deficits with inhibition might be limited to the verbal domain.

\section{SUBJECT BACKGROUND INFORMATION}

\section{Patient M.L.}

Patient M.L. is a 62-year-old right-handed male with a left-hemisphere lesion resulting from a cerebrovascular accident (CVA) in 1990. He completed 2 years of college coursework and was employed as a draftsman prior to his CVA. His lesion includes the left frontal and parietal operculum, with atrophy noted in the left temporal operculum and with mild diffuse atrophy.

M.L. has a verbal STM deficit, since his accuracy of list recall is $77 \%$ lists correct for two-word lists and $10 \%$ correct for three-word lists (for lists recalled in order). As has been reported in several studies by Martin and colleagues (Freedman \& Martin, 2001; R. C. Martin \& He, 2004; R. C. Martin \& Lesch, 1996), a number of features of M.L.'s STM deficit support the conclusion that he has a deficit in semantic retention but shows better retention of phonological information. For instance, he shows no advantage for word recall over nonword recall, whereas patients with a phonological retention deficit typically show a large advantage for words over nonwords (Martin \& Lesch, 1996). It should be noted, however, that M.L.'s retention of phonological information does not appear to be normal, since his nonword span is reduced. However, his semantic retention is more impaired than his phonological retention. Freedman and Martin (2001) computed a composite z score for performance on a number of measures tapping semantic and phonological retention. M.L.'s semantic composite retention score was -2.59 , whereas his composite phonological retention score was -0.23 . (In contrast, Patient E.A., with a phonological retention deficit, obtained a composite semantic score of 3.86 and a composite phonological score of -3.95).

M.L. demonstrates good comprehension of conversational speech on a clinical exam, but his narrative pro- duction is plagued by pausing, hesitations, word-finding difficulties, and reduced phrase length. He demonstrates no apraxia of speech, and his repetition of single words is excellent ( $96 \%$ correct).

M.L.'s difficulties with semantic retention on STM tasks and with spontaneous speech cannot be attributed to difficulty in comprehending word meanings or producing individual words. As was reported by R. C. Martin and Lesch (1996), M.L. scored above the mean for control subjects on the Peabody Picture Vocabulary Task (Dunn \& Dunn, 1981), a standardized test of word comprehension, using norms for 40-year-old participants (the highest age for which norms are available). On the Philadelphia Naming Test (Roach, Schwartz, N. Martin, Grewal, \& Brecher, 1996), which involves naming pictured objects, M.L. scored $98 \%$ correct, which was above the mean for control subjects ( $96 \%$ correct). As was reported by R. C. Martin and He (2004), he performed at a normal level of accuracy on unspeeded and speeded tasks examining living-nonliving judgments and category judgments. His reaction times (RTs) on the living-nonliving judgment were just outside the normal range but, on the category judgments, were substantially longer than those for controls. However, as was argued by R. C. Martin et al. (1994), the category judgments place some demand on semantic STM, since the subject must retain the category label while deciding whether the exemplar is a member of the category.

Despite M.L.'s excellent performance on most semantic tasks, the question is often raised as to whether his deficit in RTs on some semantic tasks might reflect a disruption of semantic knowledge representations that underlies his poor STM performance. It should be noted, however, that M.L.'s STM performance contrasts substantially with that of patients who do have severe semantic deficits - that is, semantic dementia patients who have a degenerative disorder that selectively affects semantic knowledge (see Hodges, Patterson, Oxbury, \& Funnell, 1992). In contrast to the primarily frontal damage for M.L., these patients have progressive focal atrophy of the inferotemporal cortex, which is typically more pronounced in the left hemisphere (Snowden, Goulding, \& Neary, 1989). Several such patients, who performed far below the normal range on semantic tasks, such as picture naming and word comprehension, have been assessed on their STM performance (e.g., Jefferies, Bateman, \& Lambon Ralph, 2005; McCarthy \& Warrington, 2001; Patterson, Graham, \& Hodges, 1994). Despite their severe semantic deficits, these patients' spans appear to be larger than M.L.'s. For example, the semantic dementia patient A.M. reported by Knott, Patterson, and Hodges (1997) scored more than 10 standard deviations below the control mean in accuracy of picture naming and word comprehension, yet scored $56 \%$ lists correct for four-item word list recall. M.L. scored 0\% lists correct for fouritem lists composed of similar materials. Also, the semantic dementia cases routinely perform better on word than on nonword lists, at least when the word lists are composed of words that the patient still comprehends (see 
Jefferies et al., 2005, for a discussion). Although it certainly would be valuable to compare M.L. and semantic dementia cases on the same materials, the existing evidence suggests that it would be difficult to attribute M.L.'s STM pattern to a disruption of semantic knowledge.

\section{Control Subjects}

All the control subjects were selected from a pool of older adults that regularly participate in experiments at Rice University's Brain and Language Lab. Ages ranged from 53 years to 68 years, with a mean age of 59 years $(S D=4.6)$. Not all the control subjects were available for testing in all the experiments. All the control subjects had attended at least some college.

\section{EXPERIMENTAL TASKS}

\section{Stroop Task}

Stroop interference is a classic measure of interference and executive control. We administered a Stroop task consisting of a single mixed block of neutral, congruent, and incongruent trials. We also assessed M.L.'s naming of colors outside the Stroop context, to examine the possibility that repeated naming of colors might elicit additional interference for M.L. This was necessary given other data suggesting that semantically related stimuli also induce interference in naming for M.L. (Freedman, Martin, \& Biegler, 2004). A similar interference effect during repeated naming of semantically related exemplars has been reported in healthy subjects (Damian, Vigliocco, \& Levelt, 2001; Kroll \& Stewart, 1994; Pickard, Brandon, Hodgson, Schwartz, \& Thompson-Schill, 2003). Thus, we argue that M.L.'s RTs for color naming outside the Stroop task provide a useful comparison with his naming on the neutral trials within the Stroop task. Such a comparison provides evidence on whether repeated sampling from the same semantic set causes interference in producing the appropriate color name. We also assessed whether M.L. demonstrated anomalous effects of facilitation. This was in accord with recent studies suggesting that facilitation may also reflect poor performance in the Stroop task (Kane \& Engle, 2003). Moreover, given past objections to the use of traditional difference scores in measuring Stroop interference in the literature on aging (see Verhaeghen \& De Meersman, 1998), we also employed a more conservative measure of Stroop interference, using log transforms.

\section{Method}

Materials and Procedure. A computerized Stroop task (Stroop, 1935) provided by Akira Miyake (University of Toronto, St. George) was used. The subjects named aloud the color of each stimulus (color words or rows of asterisks) as quickly as possible. A voice key was used to measure voice onset latencies. The subjects saw 72 neutral trials consisting of asterisks appearing in red, green, blue, orange, yellow, or purple. Sixty incongruent trials consisted of color words (red, green, blue, orange, yellow, or purple) that appeared in a different color (red, green, blue, orange, yellow, or purple). In addition, 12 congruent trials consisted of color words appearing in same color (red, green, blue, orange, yellow, or purple). The trials were presented in a fixed prerandomized order. All voice onset latencies beyond 2.5 standard deviations above the mean for each condition for each subject were removed for analysis. However, we also submitted untrimmed data to analysis, using log transforms.

\section{Results}

Interference was defined as the mean difference in voice onset time between incongruent trials and neutral trials. The data for patient M.L. and 10 control subjects appear in Table 1. At a group level, the controls demonstrated the expected Stroop interference effect $[M=197 \mathrm{msec}, S D=$ $62 \mathrm{msec} ; t(9)=-10.0, p<.0001]$. M.L. showed dramatically greater interference $(969 \mathrm{msec})$ than the controls did, which was 12.4 standard deviations above the mean interference effect for the controls and well outside the range $(101-279 \mathrm{msec})$. Using items as a random factor, M.L.'s interference effect was statistically significant $[t(111)=$ $-3.38, p<.001]$.

Facilitation was defined as the mean difference in voice onset time between neutral trials and congruent trials. The control participants showed no facilitation effect $[t(9)=-1.21, p=.26]$. In fact, the control subjects were $51 \mathrm{msec}$ slower to respond to congruent trials, relative to neutral trials. M.L., however, displayed substantial facilitation of $581 \mathrm{msec}$. This facilitation effect failed to reach statistical significance $[t(71)=1.27, p=.21]$; however, there were only 11 congruent trials in this task, thereby limiting statistical power.

We also calculated interference by subtracting the mean onset latencies for congruent trials from the mean onset latencies for incongruent trials. Here the difference for patient M.L. was 1,550 msec, 9.18 standard deviations above the mean for the 10 controls $(M=146 \mathrm{msec}, S D=$ $153 \mathrm{msec}$ ) and beyond the controls' range (range from $-176 \mathrm{msec}$ to $330 \mathrm{msec}$ ).

Given the concerns about comparing difference scores for individuals who show large differences in mean RTs (Verhaeghen \& De Meersman, 1998), interference was also calculated using log transforms of untrimmed data. These data are presented in Table 2. When transformed onset latencies were used, M.L.'s interference effect was 0.17 which was 1.9 standard deviations above the mean interference for the control subjects $(M=.086, S D=.043)$. Furthermore, M.L.'s interference was outside the range of the controls (range from -.009 to .14). When the same method for the incongruent-congruent effect was used, M.L.'s interference effect was .258 and 3.55 standard deviations above the control mean $(M=.057, S D=.057)$.

Table 1

Reaction Times (RTs, in Milliseconds), Interference (in Milliseconds), and Accuracy (\% Correct) on the Stroop Task for Controls and Patient M.L.

\begin{tabular}{lrrrrrrrrr}
\hline & \multicolumn{2}{c}{ Congruent } & & \multicolumn{2}{c}{ Neutral } & & \multicolumn{2}{c}{ Incongruent } & \\
\cline { 2 - 3 } Participants & RT & Acc. & & RT & Acc. & & RT & Acc. & Interference \\
\hline Controls & 828 & 98 & & 777 & 100 & & 974 & 93.7 & $197 *$ \\
Patient M.L. & 1,889 & 92 & & 2,470 & 89 & & 3,449 & 87 & $969 *$
\end{tabular}

$* p<.001$. 
Table 2

Log Transformed Reaction Time Data for the Stroop Task for Controls and Patient M.L.

\begin{tabular}{lccc}
\hline Participants & Congruent & Neutral & Incongruent \\
\hline Controls & 2.907 & 2.878 & 2.964 \\
Patient M.L. & 3.258 & 3.346 & 3.516
\end{tabular}

This effect was also beyond the range of the 10 control subjects (range from -.06 to .156). However, when log transforms were used, M.L. showed no facilitation effect. This was owing to the fact that $\log$ transforms used untrimmed data and M.L. had a single onset latency of over $11,000 \mathrm{msec}$ in the untrimmed data in the congruent condition, which skewed the mean. This trial was considered an outlier (greater than 2.5 standard deviations above the mean) in the conventional analysis of untransformed data.

Accuracy data are also reported in Table 1. The difference in accuracy between neutral and incongruent trials for the controls was statistically significant $[t(9)=3.09$, $p=.01]$, but the difference between neutral and congruent trials was not $[t(9)=-2.07, p=.068]$. There were no statistically significant differences in M.L.'s accuracy across conditions.

\section{Color Naming Outside Stroop Task}

Relative to naming in other tasks, M.L.'s naming of colors was unusually long in the Stroop task, even on neutral trials, which merely require naming of a colored row of asterisks. We suspected that the repeated naming of six color words might be sufficient to produce interference in production for M.L. That is, all color words are semantically related, and one might expect that repeated naming of words from this semantic category would lead to a high level of semantic activation for each and difficulty in selecting the correct response from competitors. This hypothesis was supported by the observation that M.L. showed interference during production when naming pairs of semantically related pictures, relative to unrelated pictures (Freedman et al., 2004). Related effects have been reported by Kroll and Stewart (1994) and Damian et al. (2001) for non-brain-damaged subjects in naming a series of pictures when the pictures were either from the same semantic category or from different categories (randomly intermixed). For example, Kroll and Stewart found that healthy subjects were $36 \mathrm{msec}$ slower to name categorized lists of pictures, relative to randomized lists of pictures. Damian et al. replicated this effect after controlling for possible confounding variables, such as visual similarity. These investigators have attributed the category interference effect to difficulty in selecting the correct response when closely related competitors have been highly activated through their recent production in the categorized lists. Selection from competitors might plausibly involve the inhibition of the competitors (Wheeldon \& Monsell, 1994). Consequently, a difficulty with inhibition for M.L. might lead to long times even on the neutral trials in the Stroop paradigm. In order to investigate this possibility, we compared M.L.'s naming of color patches in the context of naming line drawings with his color naming in the Stroop task. This allowed comparison of M.L.'s naming in a context that did not require repeated production of words from the same category.

\section{Method}

Materials and Procedure. Six colors (red, green, blue, orange, yellow, or purple) were randomly presented with 30 other pictures taken from five additional categories (totaling six categories). The five other categories were fruits/vegetables, animals, clothes, musical instruments, and transportation vehicles. There were six exemplars from each category, for a total of 36 pictures. Each image was presented on a computer monitor, and a voice key recorded voice onset latency. With exception of the color patches, all the pictures were line drawings. M.L. and 5 additional control subjects were tested on this task.

\section{Results}

The data for the naming task appear in Table 3. M.L. was $1,370 \mathrm{msec}$ faster to name colors in the naming task $(M=$ $1,100 \mathrm{msec}, S D=175.4$, range $=850-1,284 \mathrm{msec})$ compared with naming colors in the Stroop task $[M=$ $2,470 \mathrm{msec}, S D=1,269$, range $=1,075-5,712 \mathrm{msec}$; $t(149)=2.396, p=.02]$. In contrast, 5 control subjects showed no difference between naming neutral trials in the Stroop task $(M=818 \mathrm{msec}$, range $=662-1,110 \mathrm{msec})$ and naming color patches in the context of a naming task $[M=$ $804 \mathrm{msec}$, range $=636-902 \mathrm{msec} ; t(4)=0.24, p=.82]$.

\section{Discussion: Stroop Effect and Color Naming}

Using the traditional method to calculate Stroop interference (mean voice onset for incongruent trials minus mean voice onset for neutral trials), M.L. showed an exaggerated interference effect almost five times greater than the mean for the controls. However, given past objections to the use of traditional difference scores in measuring Stroop interference in the literature on aging (see Verhaeghen \& De Meersman, 1998), we chose to further examine M.L.'s Stroop performance, using a more conservative $\log$ transform. With $\log$ transformed data, M.L.'s interference effect was still beyond the range of all the

Table 3

Onset Latencies (in Milliseconds) for the

Naming Task for Controls and Patient M.L.

\begin{tabular}{|c|c|c|c|c|c|c|}
\hline$\underline{\text { Participants }}$ & Colors & Animals & $\begin{array}{c}\text { Fruits/ } \\
\text { Vegetables }\end{array}$ & Clothes & $\begin{array}{c}\text { Musical } \\
\text { Instruments }\end{array}$ & Transportation \\
\hline $\mathrm{Co}$ & 804 & 739 & 753 & 75 & 811 & 848 \\
\hline Patient M.L. & 1,100 & 900 & 964 & 1,119 & 879 & 1,048 \\
\hline
\end{tabular}


control subjects. Thus, this patient with a semantic STM deficit did show evidence of a deficit in inhibition on the Stroop task.

In addition to his exaggerated Stroop effect, M.L. also differed from the controls in showing much longer RTs for naming colors in the Stroop task (neutral trials) than for naming colors when color patches were mixed with line drawings. The controls showed no difference between naming colors in the Stroop task and naming colors mixed with line drawings in a naming task. ${ }^{1}$ As was discussed earlier, similar interference effects for the production of semantically related words in close succession have been reported for M.L. (Freedman et al., 2004) and for young normal subjects (see Damian et al., 2001; Kroll \& Stewart, 1994). M.L.'s much longer times for the neutral condition in the Stroop task than in naming color patches mixed with other items could also be attributed to a difficulty with inhibition - that is, difficulty inhibiting competitors from the same semantic category that are strongly activated because they have recently been produced on other trials.

To summarize, relative to the controls, M.L. demonstrated an exaggerated interference effect in the traditional Stroop task. Moreover, M.L. showed much longer onset latencies for color naming in the Stroop task, when compared with naming of colors outside the Stroop task. The controls did not show this effect. The longer color naming times in the context of the Stroop task could also be plausibly attributed to a difficulty with inhibitionthat is, difficulty inhibiting other color names when they were all highly activated during the Stroop task.

\section{Nonverbal Spatial Stroop Task}

A nonverbal analogue to the Stroop task was designed to further assess M.L.'s performance in tasks that required resolution of response conflict and, presumably, inhibition and to determine whether he would show similar difficulty with inhibition in verbal and nonverbal domains. For a similar task, see Clark and Brownell (1975) and Experiment 3 in Lu and Proctor (1994).

\section{Method}

Materials and Procedure. The subjects saw arrows pointing either left or right and were asked to press a key with the middle finger (for left) or the index finger (for right) of the left hand corresponding to each direction. The left hand was used because of M.L.'s mild hemiplegia on the right. The arrows appeared in one of three positions: at the left of the screen, at the right of the screen, or directly in the middle of the screen. The arrows and a fixation point appeared in a rectangular box $9.25 \mathrm{in}$. wide and $1.75 \mathrm{in}$. in height. Similar to the Stroop task, there were congruent trials (a right- pointing arrow on the right side of the display), neutral trials (either right- or left-pointing arrows appearing in the middle of the display), and incongruent trials (a left-pointing arrow appearing on the right side of the display). There were 80 trials for each condition, for a total of 240 trials. Interference was calculated by subtracting the RTs on neutral trials from RTs on incongruent trials. M.L. was administered this task twice, with several months intervening between the two administrations. The data were combined, resulting in 480 total trials. Fifteen control subjects performed this task.

\section{Results}

The results for M.L. and the 15 control subjects on the nonverbal spatial Stroop task appear in Tables 4A and 4B. The control subjects showed a 75 -msec interference effect for incongruent trials relative to neutral trials, a difference that was significant at the group level $[t(14)=10.41, p<$ $.0001, S D=27.99$, range $=27-129 \mathrm{msec}]$. M.L. showed a significant interference effect of $106 \mathrm{msec}[t(309)=$ $7.66, p<.0001]$. This interference effect was greater than the control mean, but only 1 standard deviation above the mean for controls and well within their range. M.L.'s mean RTs for both incongruent and neutral trials were also within the range for the healthy controls. There were no differences in accuracy for either the controls or M.L.

Although M.L. clearly performed at a normal level on this spatial Stroop task, the RT difference for the controls between the conflict and the neutral conditions was less than half that for the verbal Stroop task, raising the possibility that the spatial Stroop task is simply easier. However, the standard deviation of the difference scores between the two conditions was also substantially smaller in the spatial than in the verbal Stroop task. Effect sizes for RTs for the control subjects were very large for both experiments and fairly similar in magnitude (Cohen's $d=$ 3.33 for the verbal Stroop and 2.78 for the spatial Stroop tasks). (See Rosnow \& Rosenthal, 2003, for calculation of Cohen's $d$ for repeated measures.) Thus, in terms of effect size, there is little evidence that the spatial task was substantially easier for the controls.

\section{Discussion: Nonverbal Spatial Stroop Task}

On this task, selected as a nonverbal analogue to the classic Stroop task, M.L. showed performance well within the range for the control subjects. Thus, resolving response conflict in this nonverbal task did not prove to be as difficult as resolving conflict in the verbal Stroop task. Although some may argue that the task used here differs fundamentally from the classical Stroop task in many ways, it is important to note that similar tasks have traditionally been considered to represent a variation of Stroop interference (see MacLeod, 1991, for a review). The difference

Table 4A

Reaction Times (RTs, in Milliseconds) and Interference (in Milliseconds) on a Nonverbal Spatial Stroop Task for Controls and Patient M.L.

\begin{tabular}{|c|c|c|c|c|c|c|c|c|c|c|c|c|}
\hline \multirow[b]{2}{*}{ Participants } & \multicolumn{3}{|c|}{ Congruent } & \multicolumn{3}{|c|}{ Neutral } & \multicolumn{3}{|c|}{ Incongruent } & \multicolumn{3}{|c|}{ Interference } \\
\hline & $\mathrm{RT}$ & Range & $S D$ & RT & Range & $S D$ & RT & Range & $S D$ & Neut.-Incong. & Range & $S D$ \\
\hline Controls & 593 & $566-993$ & 99 & 611 & $476-880$ & 94 & 686 & $566-993$ & 101 & 75 & $27-129$ & 28 \\
\hline Patient M.L. & 585 & & & 556 & & & 662 & & & 106 & & \\
\hline
\end{tabular}


Table 4B

Accuracy (\% Correct) on a Nonverbal Spatial Stroop Task for Controls and Patient M.L.

\begin{tabular}{|c|c|c|c|c|c|c|}
\hline \multirow[b]{2}{*}{ Participants } & \multicolumn{2}{|c|}{ Congruent } & \multicolumn{2}{|c|}{ Neutral } & \multicolumn{2}{|c|}{ Incongruent } \\
\hline & Acc. & Range & Acc. & Range & Acc. & Range \\
\hline Controls & 99.7 & $97.5-100$ & 99.8 & $98.75-100$ & 99.8 & $98.75-100$ \\
\hline Patient M.L. & 100 & & 100 & & 100 & \\
\hline
\end{tabular}

in M.L.'s performance, relative to the controls, for the standard Stroop and the nonverbal spatial Stroop tasks suggests that M.L.'s deficit in inhibition may be limited to the verbal domain. His performance on the next task, the antisaccade task, provided another means of testing this possibility. Also, given that the antisaccade task is generally a difficult task, with accuracy in the range of $79 \%-88 \%$ for undergraduate subjects (Roberts et al., 1994), data from this task should provide further evidence for whether M.L.'s performance on inhibition tasks relates to the difficulty of the task or whether the task is in the verbal or the nonverbal domain.

\section{Antisaccade Task}

The antisaccade task used here was provided by Akira Miyake and was adapted from Roberts et al. (1994). Given previous studies reporting correlations between antisaccade performance and WM function, this task was of particular interest for M.L.

\section{Method}

Materials and Procedure. Each trial began with a fixation point that was presented for a variable length. Specifically, the fixation appeared for one of nine lengths spaced at 250 -msec intervals between 1,500 and 3,500 msec. Next, a cue appeared on one side of the screen, $3.4 \mathrm{in}$. from fixation, for $175 \mathrm{msec}$ (this was more brief than the cue in Miyake et al., 2000, which was $225 \mathrm{msec}$ ). The cue was followed by a target appearing on the opposite side of the screen, $3.4 \mathrm{in}$. from fixation, for $150 \mathrm{msec}$. The cue was a small black square, whereas the target was an arrow that pointed to the left, up, or to the right. After $150 \mathrm{msec}$ the target was replaced by cross-hatching to mask after images that might aid the subjects in identifying the target. Correct identification of the target required the subject to press a key corresponding to left, right, or up. In the antisaccade task, the subjects must resist making a reflexive saccade to the initial cue, in order to detect the briefly presented target on the opposite side of the screen. If the subject makes an initial saccade to the cue, the target is difficult, if not impossible, to identify, due to the brief presentation of the target. The task began with 22 practice trials, followed by 90 target trials presented in a fixed prerandomized order. M.L. was administered the antisaccade task twice over a 6-month span, and combined data from these two administrations will be reported below.

We also administered a prosaccade task with the same parameters as those described above. However, in the prosaccade task, the cue always predicted the target location. Thus, the subject was to direct an eye movement toward the cue in order to detect the target. Different control subjects were tested in the antisaccade and the prosaccade tasks.

\section{Results}

As was expected, the 12 controls were substantially less accurate on the antisaccade $(72 \%$ correct) than on the prosaccade $(97 \%$ correct) task. On the antisaccade task, M.L.'s accuracy was above the mean for the controls (M.L. $=80 \%$, control $M=72 \%$, control $S D=$ 11.8 , control range $=59 \%-94 \%)$. His mean RT $(M=$ $724 \mathrm{msec}$ ) was well within the range for the controls (control $M=771 \mathrm{msec}, S D=236$, control range = 492-1,394 msec). M.L.'s accuracy on the prosaccade task was $93 \%$ and was within the range for tested controls $(M=97 \%$, range $=93 \%-100 \%)$. M.L.'s mean RT on the prosaccade task was $521 \mathrm{msec}$, which was within range for the controls $(M=485 \mathrm{msec}, S D=80$, range $=$ 386-608 msec). Thus, M.L. performed in a normal fashion on these tasks.

\section{Discussion: Antisaccade Task}

As with the nonverbal spatial Stroop task, M.L. performed at a normal level on the antisaccade task. As was anticipated, the antisaccade task was a quite difficult task for the controls, with a mean accuracy of only $72 \%$ as compared with $97 \%$ for the prosaccade task. This $27 \%$ difference in accuracy between the two conditions was substantially larger than that between the incongruent and the neutral conditions $(6.3 \%)$ and between the incongruent and the congruent conditions $(4.3 \%)$ in the verbal Stroop task. The RT difference of $207 \mathrm{msec}$ between the antisaccade and the prosaccade tasks was also somewhat larger than that observed between the incongruent and the neutral conditions (197 msec) and between the incongruent and the congruent conditions $(126 \mathrm{msec})$ in the verbal Stroop task. Consequently, it is highly unlikely that M.L.'s excellent performance on the antisaccade task could be attributed to its being a generally easier task than the standard verbal Stroop task. Thus, the results from both the antisaccade and the spatial Stroop tasks, as compared with the standard Stroop task, provide evidence that M.L.'s difficulty with inhibition is limited to the verbal domain. This hypothesis will be further tested through use of the fourth task, the recent-negatives task.

M.L.'s normal level of performance on the antisaccade task would seem to go against findings from normal subjects indicating a relation between antisaccade performance and WM capacity (Kane et al., 2001). Further discussion of this point will be delayed until the General Discussion section.

\section{Proactive Interference: Recent-Negatives Task}

To further assess M.L.'s ability to inhibit irrelevant information in the verbal domain, we administered a task designed to elicit proactive interference in a test of STM. 
This task is of great interest, given the aforementioned experiments that have demonstrated correlations between STM performance and susceptibility to proactive interference in other memory paradigms (e.g., Chiappe et al., 2000; Rosen \& Engle, 1998).

A memory probe paradigm developed by Monsell (1978) was employed. In this recent-negatives task, the subject sees a list of items presented serially, followed by a probe. The subject responds yes or no according to whether the probe appeared in the list. A recent-negative probe trial is one in which the negative probe did not appear in the current list, but appeared in the list presented immediately before the current list. For the other negative probe trials, the negative probe did not appear in the previous list but appeared three lists back; this was considered a nonrecent negative probe. The same recent versus nonrecent manipulation was applied to the positive probes. A recent-positive trial included a probe that appeared in the current list as well as in the previous list. In a nonrecent-positive trial, the probe appeared both in the current list and in a list three trials back. The principal effect of interest in these manipulations is the contrast of recent-negative and nonrecent-negative probes; RTs are expected to be longer and accuracy lower for the recent negatives than for the nonrecent negatives. In other words, it should take longer to correctly reject a negative probe if it appeared in an immediately previous list.

Studies in which Monsell's (1978) recent-negatives paradigm (D'Esposito et al., 1999; Jonides et al. 1998) has been used have attributed these longer RTs to involuntary persistence of previously presented lists, or proactive interference. Jonides et al. (1998) assumed that an inhibitory mechanism must be necessary to overcome this involuntary persistence of items presented in previous lists that match a negative probe in the current list. The time needed to resolve this conflict is argued to result in the longer RTs for the recent-negative probes. In the past, we have attributed semantic STM deficits to overly rapid decay of semantic representations (R. C. Martin \& Lesch, 1996). If so, one would expect a patient with a semantic STM deficit to show less interference than normal from items in a previous list. On the other hand, if the deficit is in inhibiting irrelevant representations, an exaggerated interference effect would be predicted.

\section{Method}

Materials and Procedure. Three words were presented serially, followed by a probe word. Each item was drawn from a set of 16 words. Each word was presented for $750 \mathrm{msec}$ followed by a 100msec interstimulus interval. The third word was followed by $100 \mathrm{msec}$ interval, and then a row of $* * * *$ was presented for $400 \mathrm{msec}$, followed by a probe word presented for $600 \mathrm{msec}$. The probe word was immediately followed by blank screen, which remained until the subject responded. Essentially, the probe followed the last word in the series by $500 \mathrm{msec}$. The subject was instructed to respond as quickly as possible after the probe was presented. After the subject's response, the next trial began $250 \mathrm{msec}$ later with the presentation of the fixation point. There were 18 trials in each condition (recent and nonrecent conditions for both positive and negative trials). The stimuli were pre- sented in a fixed prerandomized order to form the recent and nonrecent conditions. Sixteen words were substituted for the 16-letter stimuli used in previously published research with this paradigm. (Words would ultimately allow a greater number of manipulations to be explored in this paradigm. For example, in other, unpublished studies, we have manipulated the number of unique stimuli presented in the task.)

\section{Results}

All RTs beyond 2.5 standard deviations above the mean for each condition for each subject were removed from the analysis of the control subjects and M.L. The data for M.L. and the 14 controls appear in Table 5. In an analysis of group data, the control subjects demonstrated a significant interference effect for RT $[M=91 \mathrm{msec}$; $S D=108 \mathrm{msec} ; t(13)=3.135, p=.008]$. In addition, the control subjects were significantly less accurate on recent-negative trials than on nonrecent-negative trials $[94.7 \%$ vs. $98.9 \%$ correct; $t(13)=-2.97, p=.01]$. M.L. demonstrated a substantial $(731-\mathrm{msec})$ interference effect in RT for recent versus nonrecent negatives. Although this difference failed to reach significance $[t(32)=1.36, p=$ $.18]$, M.L.'s interference effect was 5.9 standard deviations above the mean interference effect for the controls and substantially outside their range (from -74 to $337 \mathrm{msec}$ ). M.L. did show a statistically significant interference effect in accuracy. He was much worse on recent-negative trials (accuracy $=62.5 \%$ ) than on nonrecent-negative trials (accuracy $=87.5 \%$ ). This $25 \%$ difference was statistically significant $[t(46)=-2.04, p=.046]$ and far outside the range for the controls $(0 \%-13 \%)$.

We calculated interference effects, using a more conservative $\log$ transform of untrimmed data. With this method, M.L.'s interference effect (.145) was 3.12 standard deviations above the mean interference for the controls (.037) and well beyond the range for the controls (from -.027 to .09).

\section{Discussion: Recent-Negatives Task}

Although exaggerated interference effects in a patient with impaired STM at first appear paradoxical, Thompson-Schill et al. (2002) have reported another such patient. On the basis of neuroimaging findings from Jonides et al. (2000; Jonides et al., 1998) and D'Esposito et al. (1999) implicating BA 45 in the resolution of proactive interference, Thompson-Schill et al. (2002) tested a patient with a lesion that included BA 45. This patient showed exaggerated effects of proactive interference, similar to the effect reported in this article. Also of note, we have examined other patients with memory spans comparable to that of M.L. In these patients, most primarily with phonological STM deficits, we have failed to find exaggerated interference effects. One hypothesis (currently being tested by our laboratory) is that phonological STM deficits are better characterized as a rapid decay of representations in STM, whereas semantic STM deficits involve failures to inhibit representations that have been activated. 
Table 5

Reaction Times (RTs, in Milliseconds), Log Transformed RT Data, and Accuracy (\% Correct) for Recent-Negatives Task for Controls and Patient M.L.

\begin{tabular}{|c|c|c|c|c|c|c|c|c|c|c|c|c|c|c|}
\hline \multirow[b]{2}{*}{ Participants } & \multicolumn{3}{|c|}{ Recent Negative } & \multicolumn{3}{|c|}{ Nonrecent Negative } & \multicolumn{3}{|c|}{ Recent Positive } & \multicolumn{3}{|c|}{ Nonrecent Positive } & \multicolumn{2}{|c|}{$\begin{array}{c}\text { Interference } \\
\text { (Recent - Nonre- } \\
\text { cent Negatives) }\end{array}$} \\
\hline & RT & $\log \mathrm{RT}$ & Acc. & RT & Log RT & Acc. & RT & Log RT & Acc. & RT & Log RT & Acc. & RT & $\log$ RT \\
\hline Controls & 1,006 & 2.990 & $94.7 *$ & 915 & 2.953 & 98.9 & 873 & 2.933 & 98.9 & 872 & 2.932 & 98.9 & 91 & .037 \\
\hline Patient M.L. & 2,905 & 3.445 & $62.5^{*}$ & 2,174 & 3.300 & 87.5 & 1,474 & 3.223 & 100 & 1,416 & 3.136 & 96 & 731 & .145 \\
\hline
\end{tabular}

*Recent negative $<$ nonrecent negatives; $p<.05$.

\section{GENERAL DISCUSSION}

Although executive function has been a topic of much discussion in the neuropsychological literature, fewer patient studies have involved an attempt to better delineate the components of this complex cognitive capacity. Chief among these components is inhibition. The principal concern of this article was whether several tasks that are commonly assumed to require inhibition - specifically, the Stroop task, the antisaccade task, the nonverbal spatial Stroop task, and a proactive interference task - are tapping similar inhibition processes. Given the factoranalytic studies by Miyake et al. (2000) and Friedman and Miyake (2004), one might expect that a patient showing a deficit on one inhibition task, due specifically to problems with inhibition, would show deficits on other tasks loading on the inhibition factor. We were particularly interested in the antisaccade task, given that previous research has demonstrated a correlation between antisaccade performance and WM/STM performance in normal subjects.

The data presented in this article demonstrate dissociations among these tasks. Here, we have presented a patient, M.L., who had a semantic STM deficit and who showed exaggerated effects of interference on the standard color-naming Stroop task and the recent-negatives task, while demonstrating normal performance on a nonverbal spatial Stroop task and on the antisaccade task. Although the verbal/nonverbal dissociation suggested here must be considered with caution, two important points must be made. First, M.L.'s observed deficits are not likely attributable to disordered semantic representations. As is detailed in the patient's background information, M.L. performs remarkably well on word-processing tasks. His single-word comprehension and production are at a normal level in terms of accuracy, and even his RTs on semantic tasks are near normal for some tasks, although not when the task has a STM component. Thus, while the present data suggest that M.L. may have particular difficulty with maintenance and manipulation of verbal representations, these representations are largely intact. His performance contrasts with semantic dementia, cases who show much worse semantic processing but, typically, better STM (Jefferies et al., 2005).

A second issue is whether the degree of dissociation between verbal and nonverbal tasks for M.L. is much greater than might be observed in the normal population, given that the correlations among inhibition tasks for control subjects tend to be small (e.g., .18-.20 in the
Miyake et al., 2000, study). Of course, if the correlation in the normal population were zero, there would be little justification for assuming a common underlying inhibition factor in the first place. Let us assume that the correlation between the standard verbal Stroop effect and the nonverbal spatial Stroop effect is .2 for controls matched to M.L. in age and education. Given that M.L. scored 1 standard deviation above the mean in terms of his nonverbal spatial Stroop and assuming that $N=15$, the $95 \%$ confidence interval about the predicted value for the standard Stroop task, in terms of standard scores,

$$
\begin{aligned}
& =.2 \pm(2.14) \times \text { standard error of the predicted scores } \\
& =.2 \pm(2.14)(1.01) \\
& =[-1.97-2.37]
\end{aligned}
$$

M.L.'s verbal Stroop effect in terms of raw RTs was 12.4 standard deviations above the mean - clearly, outside of this range. In terms of log transforms, although his effect was within this interval when computed in terms of the difference between incongruent and neutral trials (1.9 standard deviations above the mean), it was outside this interval when computed in terms of the difference between incongruent and congruent trials (3.5 standard deviations above the mean). Assuming a .2 correlation between the standard Stroop effect and antisaccade performance, similar computations in predicting the verbal Stroop effect from antisaccade performance would show that M.L.'s verbal Stroop effect was even farther outside the $95 \%$ confidence interval, given that his RT difference between the pro- and the antisaccade tasks was only slightly greater than the mean for the controls and his error difference was smaller than the mean for the controls. Furthermore, the probability that a normal individual would show the pattern shown by M.L. would be substantially smaller than any of these individual computations when one took into account the probability of showing normal performance on both of the nonverbal tasks and performance outside the normal range on both of the verbal tasks.

Finally, the present data raise the intriguing possibility that semantic STM deficits may uniquely involve deficits of inhibition. Whereas phonological STM deficits may indeed result from rapid decay of phonological representations, semantic STM deficits may be uniquely characterized by failures of inhibition in the verbal domain. In experiments presently being conducted in our laboratory, we are attempting to establish to what extent this difficulty with inhibition applies to all verbal mate- 
rials or, perhaps, only to stimuli with a semantic component. Similar experiments are to be conducted with patients with phonological STM deficits, to determine whether further dissociations may be established.

\section{Implications for the Relation of Antisaccade Performance and WM}

Patient M.L.'s performance on the antisaccade task is especially noteworthy, given the previous research of Kane et al. (2001), Roberts et al. (1994), and Mitchell et al. (2002) relating WM ability to antisaccade performance. Specifically, Kane et al. have claimed that attentional control is central to WM performance and that performance on the antisaccade task reflects individuals' attentional control ability. Mitchell et al. described the relation in a somewhat opposite fashion-namely, that WM operations play a role in suppressing the prepotent behavioral response of attending toward the cued location. In either case, one might expect that a patient with reduced WM capacity would be impaired on the antisaccade task. Although M.L. showed deficits on verbal STM tasks, his performance was normal on the antisaccade task, which would seem to complicate these authors' claims. ${ }^{2}$ It might be argued, however, that M.L.'s normal performance on the antisaccade task is not relevant to claims about WM, since his span deficit could be attributed purely to an STM deficit, rather than to a WM deficit. That is, in the WM theory postulated by Engle, Tuholski, Laughlin, and Conway (1999), WM is composed of STM storage plus central executive function, and M.L.'s deficit is restricted to the STM storage component. Certainly, M.L.'s poorer retention of semantic than of phonological information on span tasks suggests that he does not have an overarching deficit in WM that affects all WM tasks equally. ${ }^{3}$

However, Kane and Engle and colleagues have argued that both the antisaccade task (Kane et al., 2001) and the Stroop task (Kane \& Engle, 2003) tap attentional control that is vital to the executive component of WM. The present results showed that M.L. performed normally on the antisaccade task but showed abnormally large interference on the verbal Stroop task. The findings for M.L. suggest that attentional control is not a unitary mechanism localized to the dorsolateral prefrontal cortex, as has been proposed by Kane and Engle (2002). The present data suggest that the correlations reported in these individual differences studies are not attributable to a shared neural substrate but, rather, to some other factor. This point is discussed further at the end of the General Discussion section.

\section{Implications for Organization of Executive Function and Inhibition}

Miyake et al. (2000) left open the question of whether the three target factors identified in their study would generalize to neurologically impaired subjects. They also left open the possibility that the target functions could be decomposed into more basic component pro- cesses. The present study suggests that Miyake's inhibition factor can indeed be further decomposed into finergrained functions, since the Stroop and the antisaccade tasks loaded on the same factor in Miyake et al.'s study. Of further interest, in another study in which a similar factor-analytic methodology and a number of additional inhibition tasks were used to examine the possibility of multiple inhibition factors, Friedman and Miyake (2004) reported that both the antisaccade task and the Stroop task load on the same factor, which they termed prepotent response inhibition. Here, again, data from our case study show dissociations of tasks loading on the same factor.

In addition to this dissociation among tasks that load on the same factor in Miyake et al. (2000) and a dissociation among antisaccade performance and STM performance, M.L. also shows seemingly paradoxical interference effects on tasks of proactive interference. Given his restricted memory span of 2.5 items, one might expect M.L. to show very little proactive interference. That is, if deficits of STM are conceptualized as a rapid decay of items in an STM or a WM buffer, he should show little proactive interference from preceding trials. The larger proactive interference effect suggests that M.L.'s STM deficit may be better conceptualized as a problem of persisting activation resulting from deficient inhibitory processes. Once representations are activated in M.L.'s STM, he appears to have difficulty in subsequently suppressing these representations. This is also consistent with the intrusions that M.L. produces during serial recall tasks.

The dissociation between verbal and nonverbal tasks reported here are consistent with a recent fMRI study demonstrating dissociable networks for the control of verbal and visuospatial tasks (Stephan et al., 2003). In this study, control processes in a verbal letter detection task were reported to preferentially activate a network in the left hemisphere, whereas control processes involved in a visuospatial task (with the same stimuli) preferentially activated a network in the right hemisphere. More important, the network activated in the verbal letter task involved a left region of the anterior cingulate and the left inferior frontal gyrus. In contrast, the visuospatial task activated a network involving the right anterior cingulate and the intraparietal sulcus. The authors suggested that both content and control processes for any task might prove to be lateralized in the same hemisphere. Thus, M.L.'s damage to the left inferior frontal gyrus undermines control processes for verbal information, and his spared right hemisphere leaves his performance of visuospatial tasks unimpaired.

The dissociations presented here also support recent neuroimaging data by Nelson, Reuter-Lorenz, Sylvester, Jonides, and Smith (2003) indicating different areas of activation for inhibition of previously executed motor responses and inhibition of previously presented mnemonic stimuli. Our data would appear to support such a dissociation. For example, M.L. shows no deficit on the nonverbal spatial Stroop task, which requires resolution of motor response conflict, but shows exaggerated inter- 
ference on the recent negatives task, which requires inhibition of previously presented items in memory. This further suggests the need for finer-grained distinctions in models of executive function.

Although the present data serve as interesting tests of factor-analytic studies of executive function, the behavioral dissociations correspond with much of the neuroimaging literature in which these specific tasks have been examined. First, it is interesting to note that M.L.'s lesion is located in the inferior frontal gyrus, including the frontal and parietal opercula. This area is very near the site of a lesion reported in Patient R.C. in Thompson-Schill et al. (2002), who showed similar effects of exaggerated proactive interference. Also, this corresponds with the lesions of patients with semantic STM deficits reported by R. C. Martin and colleagues (R. C. Martin \& Freedman, 2001; Romani \& Martin, 1999). This leads to the speculation that, perhaps, semantic STM deficits may be uniquely characterized by deficits of inhibition and interference.

Neuroimaging data are also potentially useful in explaining the behavioral dissociations among the antisaccade and the proactive interference tasks reported in this article. For example, as was mentioned previously, imaging studies have reported activations in BA 45 for the proactive interference task (D'Esposito et al., 1999; Jonides et al., 2000; Jonides et al., 1998). However, a recent neuroimaging study in which the antisaccade task was used (Curtis and D'Esposito, 2003) reported activity in the presupplementary eye fields in the preparatory period before execution of saccades away from a cue. This area is located along the medial wall of the frontal cortex near the interhemispheric fissure-well superior and medial to the areas reported in neuroimaging tasks of the proactive interference task (Jonides et al., 1998), as well as to M.L.'s lesion in the inferior frontal gyrus. On the basis of neuroimaging data, it seems less than surprising that we observed the dissociations reported in the present study (see also Jonides, Badre, Curtis, Thompson-Schill, \& Smith, 2002).

The present data illustrate the necessity of recognizing distinctions among tasks assumed to tap inhibition and the utility of patient studies in complementing neuroimaging data in cognitive neuroscience. Whereas carefully designed neuroimaging studies have proven invaluable in elucidating the neural substrate of specific cognitive processes, patient studies are useful in corroborating conclusions concerning the functional significance of these data.

\section{Discrepancies Between Factor-Analytic Studies and Patient Data}

Finally, we will address why factor analytic studies and patient studies might yield different conclusions with regard to the organization of executive function. One alternative would be to simply claim that the data from 1 subject, despite the degree of dissociation, do not constitute strong enough data to invalidate the results from a large number of normal subjects. However, we offer these data as a first attempt to assess the generalizability of Miyake et al.'s (2000) data to "neuropsycho- logical populations," as was suggested by Miyake et al. (p. 91). Of course, further case studies that replicate this dissociation would be important to obtain. In fact, we do not intend to claim that the present data are incompatible with the factor-analytic studies. We do suggest, however, that the present data elucidate the possible source of shared variance among these tasks. To be explicit, on the basis of M.L.'s data, shared variance does not appear to be due to all inhibition tasks sharing a common neural substrate. Although Miyake et al. and Friedman and Miyake (2004) do not claim that a common neural substrate is the source of shared variance in their studies, we feel that it is a reasonable possibility to address.

If a shared neural substrate is not the source of the shared variance, what might be? One possibility is that variations in performance among normal subjects result from variations in the level of neurotransmitters, such as dopamine and norepinephrine. Neurotransmitter function might affect the operation of a number of different frontal brain areas. Thus, even though different cortical areas are involved in different executive function tasks, the shared variance among tasks observed for normal subjects might relate to activity of a small number of neurotransmitters. Consequently, tasks supported by diverse cortical areas may load on the same factor because of intercorrelations stemming from shared dependence on neurotransmitter function. The different neural substrates involved in the different tasks could be differentially affected by brain damage - thus explaining the dissociations reported here. This hypothesis is appealing, given that deficits of executive function have often been reported with Parkinson's disease and schizophrenia, both conditions being characterized by abnormal dopamine function. Although dopamine function and cognitive performance have been examined in both humans and nonhuman primates, the relationship between dopamine and prefrontal functioning is complex. Effects may differ depending on the subreceptors examined and the tasks performed (for a review, see Arnsten \& Robbins, 2002). However, psychopharmacological studies in which the cognitive effects of dopamine agonists and antagonists in humans are examined could begin to address these issues in the future (see Kimberg \& D'Esposito, 2003, for a recent example of a psychopharmacological approach).

\section{REFERENCES}

Arnsten, A. F.T., \& RobBins, T. W. (2002). Neurochemical modulation of prefrontal cortical function in humans and animals. In D. T. Stuss \& R. T. Knight (Eds.), Principles of frontal lobe function (pp. 51-84). New York: Oxford University Press.

Chiappe, P., Hasher, L., \& Siegel, L. S. (2000). Working memory, inhibitory control, and reading disability. Memory \& Cognition, 28, 8-17.

CLARK, H. H., \& BRownell, H. H. (1975). Judging up and down. Journal of Experimental Psychology: Human Perception \& Performance, 1,339-352.

CURTIS, C. E., \& D'Esposito, M. (2003). Success and failure suppressing reflexive behavior. Journal of Cognitive Neuroscience, 15, 409-418.

Damian, M. F., Vigliocco, G., \& LeVelt, W. J. M. (2001). Effects of semantic context in the naming of pictures and words. Cognition, 81, B77-B86.

D’Esposito, M., Postle, B. R., Jonides, J., \& Smith, E. E. (1999). The 
neural substrate and temporal dynamics of interference effects in working memory as revealed by event-related fMRI. Proceedings of the National Academy of Sciences, 96, 7514-7519.

DunN, L. M., \& DunN, L. M. (1981). Peabody Picture Vocabulary Test-Revised. Circle Pines, MN: American Guidance Service.

Engle, R. W., Tuholski, S. W., Laughlin, J. E., \& Conway, A. R. A. (1999). Working memory, short-term memory, and general fluid intelligence: A latent-variable approach. Journal of Experimental Psychology: General, 128, 309-331.

FreEDMAN, M. L., \& MARTIN, R. C. (2001). Dissociable components of short-term memory and their relation to long-term learning. Cognitive Neuropsychology, 18, 193-226.

Freedman, M. L., MARTin, R. C., \& Biegler, K. (2004). Semantic relatedness effects in conjoined noun phrase production: Implications for the role of short-term memory. Cognitive Neuropsychology, 21, 245-265.

FrIEDMAN, N. P., \& MiYAKE, A. (2004). The relations among inhibition and interference control functions: A latent variable analysis. Journal of Experimental Psychology: General, 133, 101-135.

Hodges, J. R., Patterson, K., Oxbury, S., \& FunNell, E. (1992). Semantic dementia: Progressive fluent aphasia with temporal lobe atrophy. Brain, 115, 1783-1806.

Jefferies, E., Bateman, D., \& Lambon Ralph, M. A. (2005). A semantic contribution to nonword recall? Evidence for intact phonological processes in semantic dementia. Cognitive Neuropsychology, 22, 183-212.

JoNides, J., BADRE, D., Curtis, C., Thompson-Schill, S. L., \& SMith, E. E. (2002). Mechanisms of conflict resolution in prefrontal cortex. In D. T. Stuss \& R. T. Knight (Eds.), Principles of frontal lobe function (pp. 233-245). New York: Oxford University Press.

Jonides, J., Marshuetz, C., Smith, E. E., Reuter-Lorenz, P. A., Koepre, R. A., \& Hartley, A. (2000). Age differences in behavior and PET activation reveal differences in interference resolution in verbal working memory. Journal of Cognitive Neuroscience, 12, 188-196.

Jonides, J., Smith, E. E., Marshuetz, C., Koeppe, R. A., \& ReuterLoRENZ, P. A. (1998). Inhibition in verbal working memory revealed by brain activation. Proceedings of the National Academy of Sciences, 95, 8410-8413.

Kane, M. J., Bleckley, M. K., Conway, A. R. A., \& Engle, R. W. (2001). A controlled-attention view of working-memory capacity. Journal of Experimental Psychology: General, 130, 169-183.

KANE, M. J., \& ENGLE, R. W. (2002). The role of prefrontal cortex in working-memory capacity, executive attention, and general fluid intelligence: An individual-differences perspective. Psychonomic Bulletin \& Review, 9, 637-671.

KANE, M. J., \& ENGLE, R. W. (2003). Working-memory capacity and control of attention: The contributions of goal neglect, response competition, and task set to Stroop interference. Journal of Experimental Psychology: General, 132, 47-70.

KIMBERG, D. Y., \& D'Esposito, M. (2003). Cognitive effects of the dopamine receptor agonist pergolide. Neuropsychologia, 41, 1020-1027.

Knott, R., Patterson, K., \& Hodges, J. R. (1997). Lexical and semantic binding effects in short-term memory: Evidence from semantic dementia. Cognitive Neuropsychology, 14, 1165-1218.

Kroll, J. F., \& SteWART, E. (1994). Category interference in translation and picture naming: Evidence for asymmetric connections between bilingual memory presentations. Journal of Memory \& Language, 33, 149-174.

Lu, C.-H., \& Proctor, R. W. (1994). Processing of an irrelevant location dimension as a function of the relevant stimulus dimension. Journal of Experimental Psychology: Human Perception \& Performance, 20, 286-298.

MACLEOD, C. M. (1991). Half a century of research on the Stroop effect: An integrative review. Psychological Bulletin, 109, 163-203.

Martin, N., \& SAFFran, E. M. (1997). Language and auditory-verbal short-term memory impairments: Evidence for common underlying processes. Cognitive Neuropsychology, 14, 641-682.

Martin, R. C., \& Freedman, M. (2001). The neuropsychology of ver- bal working memory: The ins and outs of phonological and lexicalsemantic retention. In H. L. Roediger, III, J. S. Nairne, I. Neath, \& A. M. Surprenant (Eds.), The nature of remembering: Essays in honor of Robert G. Crowder (pp. 331-349). Washington, DC: American Psychological Association Press.

MARTIN, R. C., \& HE, T. (2004). Semantic short-term memory and its role in sentence processing: A replication. Brain \& Language, 89, 76-82.

Martin, R. C., \& LESCH, M. F. (1996). Associations and dissociations between language impairment and list recall: Implications for models of short-term memory. In S. Gathercole (Ed.), Models of shortterm memory (pp. 149-178). Hove, U.K.: Erlbaum.

Martin, R. C., Shelton, J. R., \& Yaffee, L. S. (1994). Language processing and working memory: Neuropsychological evidence for separate phonological and semantic capacities. Journal of Memory \& Language, 33, 83-111.

MAY, C. P., HASHER, L., \& KANE, M. J. (1999). The role of interference in memory span. Memory \& Cognition, 27, 759-767.

McCARTHY, R. A., \& WARrington, E. K. (2001). Repeating without semantics: Surface dysphasia? Neurocase, 7, 77-87.

Mitchell, J. P., Macrae, C. N., \& Gilchrist, I. D. (2002). Working memory and the suppression of reflexive saccades. Journal of $\mathrm{Cog}_{-}$ nitive Neuroscience, 14, 95-103.

Miyake, A., Friedman, N. P., Emerson, M. J., Witzki, A. H., HowERTER, A., \& WAGER, T. D. (2000). The unity and diversity of executive functions and their contributions to complex "frontal lobe" tasks: A latent variable analysis. Cognitive Psychology, 41, 49-100.

Monsell, S. (1978). Recency, immediate recognition memory, and reaction time. Cognitive Psychology, 10, 465-501.

Nelson, J. K., ReUTER-LorenZ, P. A., SYlVester, C.-Y. C., Jonides, J., \& SMITH E. E. (2003). Dissociable neural mechanisms underlying response-based and familiarity-based conflict in working memory. Proceedings of the National Academy of Sciences, 100, 11171-11175.

Patterson, K., Graham, N., \& Hodges, J. R. (1994). The impact of semantic memory loss on phonological representations. Journal of Cognitive Neuroscience, 6, 57-69.

PiCKard, J., Brandon, M., Hodgson, C., Schwartz, M., \& ThompsonSCHILl, S. (2003, March). Facilitating and interfering effects of context on picture naming. Poster presented at the 10th Annual Meeting of the Cognitive Neuroscience Society, New York.

Roach, A., Schwartz, M. F., Martin, N., Grewal, R. S., \& BreCHER, A. (1996). The Philadelphia Naming Test: Scoring and rationale. Clinical Aphasiology, 24, 121-134.

Roberts, R. J., Hager, L. D., \& Heron, C. (1994). Prefrontal cognitive processes: Working memory and inhibition in the antisaccade task. Journal of Experimental Psychology: General, 123, 374-393.

RoMANI, C., \& MARTIN, R. [C.] (1999). A deficit in the short-term retention of lexical-semantic information: Forgetting words but remembering a story. Journal of Experimental Psychology: General, 128, 56-77.

Rosen, V. M., \& EnGLE, R. W. (1997). The role of working memory capacity in retrieval. Journal of Experimental Psychology: General, 126, 211-227.

RoSEN, V. M., \& ENGLE, R. W. (1998). Working memory capacity and suppression. Journal of Memory \& Language, 39, 418-436.

Rosnow, R. L. \& RosenthaL, R. (2003). Effect sizes for experimenting psychologists. Canadian Journal of Experimental Psychology, 57, 221-237.

Rouleau, I., Imbault, H., Laframboise, M., \& Bédard, M. A. (2001). Pattern of intrusions in verbal recall: Comparison of Alzheimer's disease, Parkinson's disease, and frontal lobe dementia. Brain \& Cognition, 46, 244-249.

SNOWDEN, J. S., Goulding, P. J., \& NeARY, D. (1989). Semantic dementia: A form of circumscribed cerebral atrophy. Behavioral Neurology, 2, 167-182.

Stephan, K. E., Marshall, J. C., Friston, K. J., Rowe, J. B., Ritzl, A., ZILLES, K., \& FINK, G. R. (2003). Lateralized cognitive processes and lateralized task control in the human brain. Science, 301, 384-386.

STroop, J. R. (1935). Studies of interference in serial verbal reactions. Journal of Experimental Psychology, 18, 643-662. 
Thompson-Schill, S. L., D’Esposito, M., Aguirre, G. K., \& Farah, M. J. (1997). Role of left inferior prefrontal cortex in retrieval of semantic knowledge: A re-evaluation. Proceedings of the National Academy of Sciences, 94, 14792-14797.

Thompson-Schill, S. L., Jonides, J., Marshuetz, C., Smith, E. E., D’Esposito, M., Kan, I. P., KNight, R. T., \& Swick, D. (2002). Effects of frontal lobe damage on interference effects in working memory. Cognitive, Affective, \& Behavioral Neuroscience, 2, 109-120.

Verhaeghen, P., \& De Meersman, L. (1998). Aging and Stroop effect: A meta-analysis. Psychology \& Aging, 13, 120-126.

WHEELDON, L. R., \& MONSELL, S. (1994). Inhibition of spoken word production by priming a semantic competitor. Journal of Memory \& Language, 33, 332-356.

Whitney, P., ARnett, P. A., Driver, A., \& Budd, A. (2001). Measuring central executive functioning: What's in a reading span? Brain \& Cognition, 45, 1-14.

ZACKS, R. T., \& HASHER, L. (1994). Directed ignoring: Inhibitory regulation of working memory. In D. Dagenbach \& T. H. Carr (Eds.). Inhibitory processes in attention, memory, and language (pp. 241-264). San Diego: Academic Press.

\section{NOTES}

1. The failure to find longer times in the neutral condition of the Stroop task than in the naming of color patches mixed with other items for the controls could not be considered a failure to replicate the find- ings of Kroll and Stewart (1994) or Damian et al. (2001) with older subjects. There were many differences between the two conditions in terms of the stimuli presented (rows of colored XXXs vs. color patches), numbers of practice trials, numbers of items, and so forth.

2 . It should be noted that some previous research in which the antisaccade task has been used has employed other measures of performancenamely, eye movement data collected by eye-tracking equipment. Although we have not incorporated eye movement data into this study, we feel confident in our assessment of M.L.'s antisaccade performance on the basis of target identification. Previous research by Roberts et al. (1994) has demonstrated that target identification data parallel data from eye movements and that both measures are similarly affected by demands of dual-task methodologies. Therefore, we suspect that M.L. would show similar performance, relative to controls, if eye movement data were collected. In fact, M.L.'s accuracy is comparable to the mean for young controls reported in Table 2 in Roberts et al. This was the case even though we used a shorter cue, a modification made by Miyake to increase variance, which presumably made the task more difficult.

3. A reviewer commented that M.L. could be tested on some spatial WM task to provide evidence on whether he has a generalized WM deficit. We predict that he would do well on such a task, but such testing has yet to be carried out.

(Manuscript received March 11, 2004; revision accepted for publication July 2, 2004.) 